\title{
Electronic and nonlinear optical properties of doped/undoped graphene via coronene based models: a DFT study
}

\author{
M. El Masfioui, S. Bahsine, A. Elbiyaali, F. Allali \\ Fundamental and Applied physics Laboratory-Safi physics department, Polydisciplanary Faculty, Cadi Ayyad University, BP \\ 4162, Sidi Bouzid, Safi, Morocco. \\ elmasfiouimohamed1@gmail.com
}

KEYWORDS

\section{DFT, Graphene}

Coronene, doping

Nonlinear optical properties

Electronic properties

Polarizability

Hyperpolarizability

\begin{abstract}
Coronene $\mathrm{C}_{24} \mathrm{H}_{12}$ can be considered as graphene nanoflakes. This aromatic hydrocarbon allows for detailed calculations and the rapid evaluation of a variety of doped structures while maintaining the periodic aromaticity of graphene. In this study, through density functional theory (DFT) at the B3LYP (BECKE 3-PARAMETER LEE-YANG-PARR) functional with a 6-31G (d) basis set, we have investigated the effects of substituting carbon atoms with $\mathrm{B}, \mathrm{N}$, and $\mathrm{O}$ on the electronic structure, linear and nonlinear optical properties of graphene.

Therefore, we found that the bandgap varies as a function of the considered structure.
\end{abstract}

\section{Introduction}

In the past few years, especially since 2010, the two physicists Geim and Novoselov have been awarded the Nobel Prize for Physics for discovering the existence of an important substance [1]. Material can create a major revolution in our daily. This material is graphene "for gr oundbreaking experiments regarding the two-

dimensional material graphene". Why is it so important? As a two-dimensional material with magical properties in terms of hardness and conductivity [2], we also talked about flexibility $[3,4,5]$. Not only that but because it only contains carbon atoms that are abundant in nature, it can be used in various fields, whether it involves medical or industrial, etc.

With this discovery related to graphene, the carbon family has been completed, and it plays a variety of roles in promoting our lives, from carbon, through graphite, to diamonds.

As for the application of graphene, we may find it in electronic devices because it is a completely different semiconductor material from all other materials because it has no ribbon. The barrier between the valence band and the conduction band (zero bandgaps) [6], which gives us a lot of electron movement and therefore a high conductivity, we are talking about mobility with a value of $15000 \mathrm{~cm}^{2} \cdot \mathrm{v}^{-1} \cdot \mathrm{s}-1$ [7], which may be the lowest in the field-effect transistor it can also be used in solar cells in gas sensors, especially in receiving light.

There are two types of methods to extract and produce graphene. We know that graphene is a single-layer carbon monad that forms a hexagon, such as a bee ring, the first type, or so-called top-down [8]. This method mainly relies on exfoliating and isolating the graphite layer until we finally get a very thin layer called graphene. In the method that relies on this process, we mentioned mechanical as well as chemical exfoliation.

The second type is called bottom-up. Unlike the first type, bottom-up occurs by forming a network of carbon atoms until graphene is obtained. Among the ways this process depends, there is Chemical Vapor Deposition (CVD) [9].

However, due to the number and diversity of these processes and methods, only a very small amount of graphene can be extracted, and it was only regarded as samples and it was considered very expensive. From here we will start our purity, which will be the basis of 
the material coronene. We will increase the number of carbon atoms in a honeycomb like a manner.

The present work investigates the properties of $\mathrm{C}_{24} \mathrm{H}_{12}, \mathrm{C}_{54} \mathrm{H}_{18}, \mathrm{C}_{18} \mathrm{~N}_{6} \mathrm{H}_{12}, \mathrm{C}_{24} \mathrm{H}_{12} \mathrm{COOH}^{-}$and $\mathrm{C}_{18} \mathrm{~B}_{6} \mathrm{H}_{12}$. to study the electronic properties of graphene. This kind of molecule enables us to achieve the same magical properties of this latter. However, we demonstrated the linear and nonlinear optical properties of doped and undoped grapheme using DFT calculations, of the maximum doping rate of typical coronene in the goal to several applications.

\section{Computational Methods}

In this work, on one hand, we calculated a set of properties of five molecules that depend on the coronene molecule which are $\mathrm{C}_{24} \mathrm{H}_{12}, \mathrm{C}_{54} \mathrm{H}_{18}, \quad \mathrm{C}_{18} \mathrm{~N}_{6} \mathrm{H}_{12}$, $\mathrm{C}_{24} \mathrm{H}_{12} \mathrm{COOH}^{-}$and $\mathrm{C}_{18} \mathrm{~B}_{6} \mathrm{H}_{12}$. As for how to calculate these features and characteristics, the DFT method is an ideal choice for this work, in addition to choosing the B3LYP functional with a 6-31G(d) basis set. Except for the Gauss-View, all these tools exist in the Gaussian v09.
All of these are for studying nonlinear optical (NLO), linear and electronic characteristics, become a good key in several applications, such as photovoltaics telecommunications, information storage, light-emitting diodes (LEDs), dynamic image processing, field effects Transistor (FET) optical switches, and other laser devices [10].

On the other hand, to calculate nonlinear optics (NLO) properties, we use the DFT method. As for the polarization tensor and hyperpolarization $\left(\alpha_{x x}, \alpha_{x y}, \alpha_{y y}\right.$, $\alpha_{x z}, \alpha_{y z}, \alpha_{z z}$ and $\beta_{x x x}, \beta_{x y}, \beta_{x y y}, \beta_{y y y}, \beta_{x x z}, \beta_{x y z} ; \beta_{y y z}$, $\beta_{x z z}, \beta_{y z z}, \beta_{z z z}$ ), they can be determined by the Gaussian frequency job output file, but the values obtained are actually in atomic units (a.u), so we must change them to electronic units (esu).

Polarizability average $\langle\alpha\rangle$, polarizability anisotropy $\Delta \alpha$, second Hyperpolarizability $\langle\gamma\rangle$, the average value of the first hyperpolarizability $\langle\beta\rangle$ can be calculated using the following formula $[11,12,13]$.

$$
\begin{gathered}
<\alpha>=1 / 3\left(\alpha_{x x}+\alpha_{y y}+\alpha_{z z}\right) \\
<>=\left[\left(\text { xxx }_{\mathrm{xyy}}+{ }_{\mathrm{xzz}}\right)^{2}+\left(\mathrm{yyy}_{\mathrm{yzz}}+\mathrm{yxx}^{2}+\left(\mathrm{zzz}_{\mathrm{zxx}}+{ }_{\mathrm{zyy}}\right)^{2}\right]^{1 / 2}\right. \\
\Delta \alpha=\frac{1}{\sqrt{2}}\left(\left(\alpha_{\mathrm{xx}}-\alpha_{\mathrm{yy}}\right)^{2}+\left(\alpha_{\mathrm{yy}}-\alpha_{\mathrm{zz}}\right)^{2}+\left(\alpha_{\mathrm{zz}}-\alpha_{\mathrm{xx}}\right)^{2}+6 \alpha_{\mathrm{xx}}^{2}+6 \alpha_{\mathrm{yy}}^{2}+6 \alpha_{\mathrm{zz}}^{2}\right)^{1 / 2} \\
\gamma=1 / 5\left\{\gamma_{\mathrm{xxxx}}+\gamma_{\mathrm{yyyy}}+\gamma_{\mathrm{zzzz}}+2\left[\gamma_{\mathrm{xxyy}}+\gamma_{\mathrm{xxz}}+\gamma_{\mathrm{yyzz}}\right]\right\}
\end{gathered}
$$

\section{Results and discussion}

\section{i) Molecular Geometry Optimization}

In this study, we utilized a few molecules exuding from the base molecule, which is coronene $\mathrm{C}_{24} \mathrm{H}_{12}$, and we moreover expanded the number of carbons $\mathrm{C}_{54} \mathrm{H}_{18}$. After that, we doped coronene with heterogeneous atoms, such as: boron (B) $C_{18} B_{6} H_{12}$, nitrogen (N) $\mathrm{C}_{18} \mathrm{~N}_{6} \mathrm{H}_{12}$, Oxygen
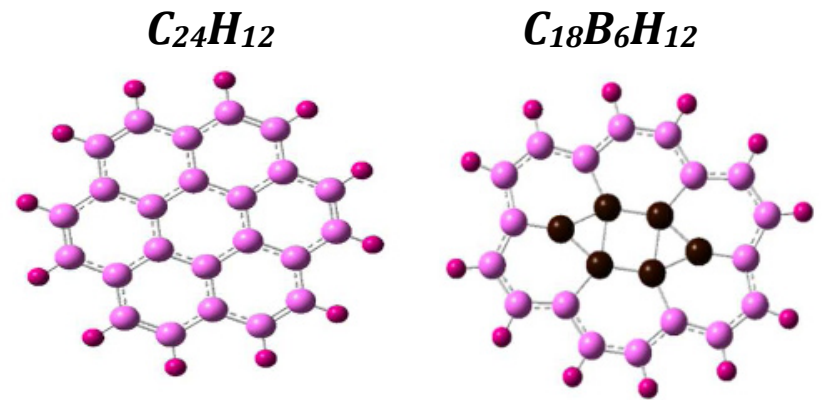

(O) $\mathrm{C}_{24} \mathrm{H}_{12} \mathrm{COOH}^{-}$. This study was done using B3LYP functional using $6-31 \mathrm{G}$ basis set to obtain fully optimized structures of undoped $\mathrm{C}_{24} \mathrm{H}_{12}, \mathrm{C}_{54} \mathrm{H}_{18}$, and doped $\mathrm{C}_{18} \mathrm{~N}_{6} \mathrm{H}_{12}, \quad \mathrm{C}_{24} \mathrm{H}_{12} \mathrm{COOH}^{-}$and $\mathrm{C}_{18} \mathrm{~B}_{6} \mathrm{H}_{12}$ (Figure 1).
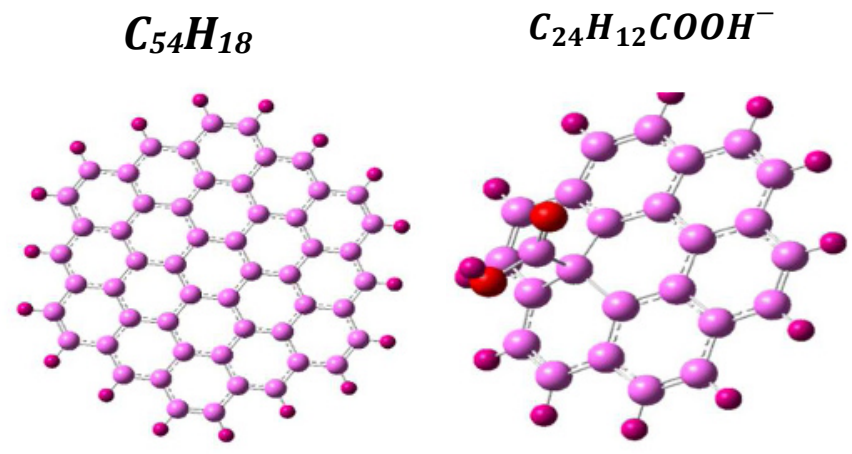


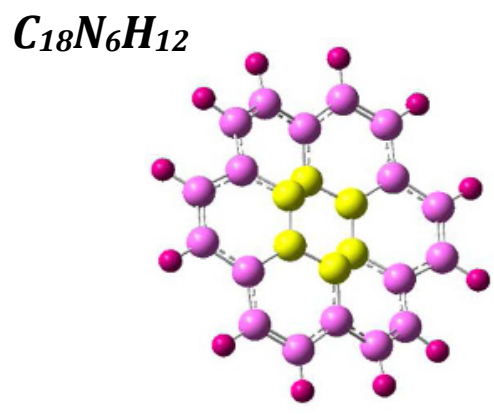

Boron
Carbone
Nitrogen
Oxygen
Hydrogen

Fig 1. Optimized molecules of (coronene), (circumcoronene), doped to Nitrogen $\left(C_{18} N_{6} H_{12}\right)$ and doped to Oxygen $\left(\mathrm{C}_{24} \mathrm{H}_{12} \mathrm{COOH}^{-}\right)$and doped to Boron $\left(\mathrm{C}_{18} \mathrm{~B}_{6} \mathrm{H}_{12}\right)$ with B3LYP/6-31G(d) basis set.

\section{ii) Electronic properties}

The energy gap is the difference between LUMO (Lowest Unoccupied Molecular Orbital) and HOMO (Highest Occupied Molecular Orbital) [14]. This parameter is very important for explanation of structural stability and reactivity. The table 1 gives the specific value of each LUMO and HOMO, as well as we allow calculating the value of the energy of the gap of each molecule [15]. the DFT method, using B3LYP/6-31 G(d). However, we have calculated the energy gap of HOMO, LUMO for five molecules, these values are partly close, so the minimum energy gap of the $C_{18} B_{6} H_{12}$ molecule is $1.39 \mathrm{eV}$, and the energy gap of the $C_{18} \mathrm{~N}_{6} \mathrm{H}_{12}$ molecule is $1.63 \mathrm{eV}$. These last values for both $C_{18} B_{6}$ $\mathrm{H}_{12}$ and $\mathrm{C}_{18} \mathrm{~N}_{6} \mathrm{H}_{12}$ decrease compared to the values obtained from undoped $\mathrm{C}_{24} \mathrm{H}_{12}$.

\begin{tabular}{|c|c|c|c|c|c|c|}
\hline Compound & HOMO (eV) & LUMO (eV) & $E_{g}=L-H(e V)$ & $\begin{array}{c}\text { E }_{\mathrm{g}: \text { other }} \\
\text { works }(\mathrm{eV})\end{array}$ & $\begin{array}{c}\text { The margin } \\
\text { of error }(\mathrm{eV})\end{array}$ & \\
\hline $\mathrm{C}_{54} \mathrm{H}_{18}$ & $-4,88$ & $-1,99$ & 2,88 & 2,86 & 0,02 & \multirow[t]{3}{*}{ [16] } \\
\hline $\mathrm{C}_{24} \mathrm{H}_{12}$ & $-5,45$ & $-1,41$ & 4,04 & 4,01 & 0,03 & \\
\hline$\left[\mathrm{C}_{24} \mathrm{H}_{12} \mathrm{COOH}\right]^{-}$ & $-0,17$ & 2,2 & 2,36 & 2,35 & 0,01 & \\
\hline $\mathrm{C}_{18} \mathrm{~N}_{6} \mathrm{H}_{12}$ & $-3,58$ & $-1,95$ & 1,63 & 1,27 & 0,36 & \multirow[t]{2}{*}[17]{} \\
\hline $\mathrm{C}_{18} \mathrm{~B}_{6} \mathrm{H}_{12}$ & $-5,17$ & $-3,78$ & 1,39 & 0,91 & 0,48 & \\
\hline
\end{tabular}

Table 1 shows the values we have obtained through

Table 1. Calculated Energy values of $\mathrm{C}_{24} \mathrm{H}_{12}, \mathrm{C}_{54} \mathrm{H}_{18}$, $\left[\mathrm{C}_{24} \mathrm{H}_{12} \mathrm{COOH}\right]^{-}, \mathrm{C}_{18} \mathrm{~N}_{6} \mathrm{H}_{12}$, and $\mathrm{C}_{18} \mathrm{~B}_{6} \mathrm{H}_{12}$ using DFT and B3LYP functional with 6-31G(d) basis set.

The coronene $\mathrm{C}_{24} \mathrm{H}_{12}$ whose carbon number is not even added with other atoms gives us great value, its value is $4.04 \mathrm{eV}$. This result is comparable to those obtained in the literature, it confirms our method used . Hence, $\mathrm{C}_{18} \mathrm{~B}_{6} \mathrm{H}_{12}$ and $\mathrm{C}_{18} \mathrm{~N}_{6} \mathrm{H}_{12}$ are great conductors compared to the rest of the molecules. Comparing these values with the results of several other fields, we find that the values of $\mathrm{C}_{24} \mathrm{H}_{12}, \mathrm{C}_{54} \mathrm{H}_{18}$, and $\mathrm{C}_{24} \mathrm{H}_{12} \mathrm{COOH}^{-}$are almost equal, while for $\mathrm{C}_{18} \mathrm{~N}_{6} \mathrm{H}_{12}$ and $\mathrm{C}_{18} B_{6} \mathrm{H}_{12}$, and we found that the obtained results are slightly larger, because we used different base. We note also that the estimation of the gap energy has changed drastically from the value $4.04 \mathrm{eV}$ for the coronene molecule, which is the premise of our work, which the obtained value close to $1.39 \mathrm{eV}$ fo $\mathrm{r}$ the $\mathrm{C}_{18} B_{6} H_{12}$ molecule. The most reduced esteem of the gap energy has diminished altogether, but usuall futile because the esteem of the gap energy within the graphene is zero, here remains the plausibility that if we increment the number of carbons by taking after the same arrangement of its hexagonal shape,we are going definitely get the esteem of the gap energy of zero, and typically what recognizes graphene because it encompasses an exceptionally conductivity

\section{HOMO}

$\mathrm{C}_{24} \mathrm{H}_{12}$

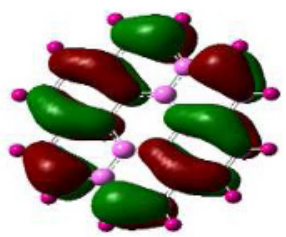

$E_{g}=4,04 \mathrm{eV}$

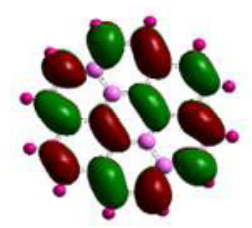




\section{$\mathrm{C}_{54} \mathrm{H}_{18}$}

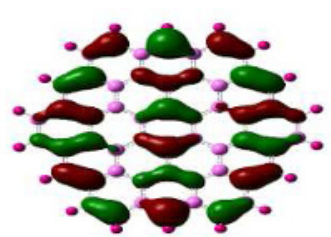

$\mathrm{E}_{\mathrm{g}}=2,88 \mathrm{eV}$
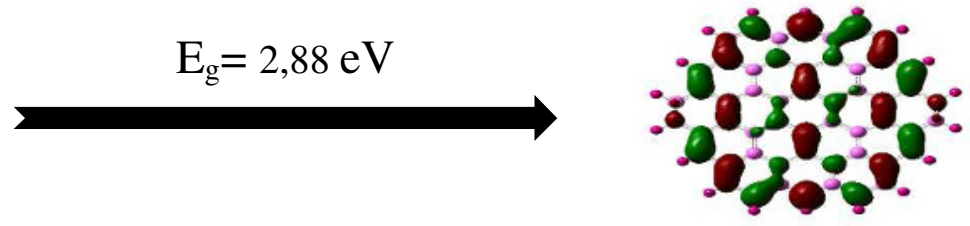

\section{$\mathrm{C}_{24} \mathrm{H}_{12} \mathrm{COOH}^{-}$}
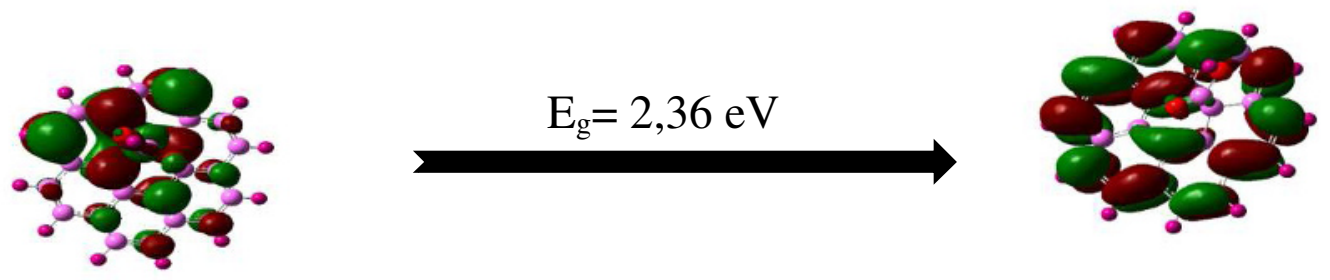

\section{$\mathrm{C}_{18} \mathrm{~N}_{6} \mathrm{H}_{12}$}
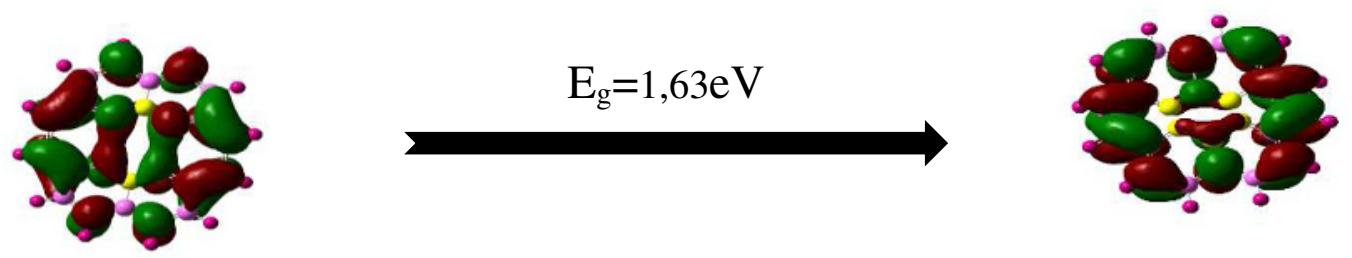

\section{$\mathrm{C}_{18} \mathrm{~B}_{6} \mathrm{H}_{12}$}
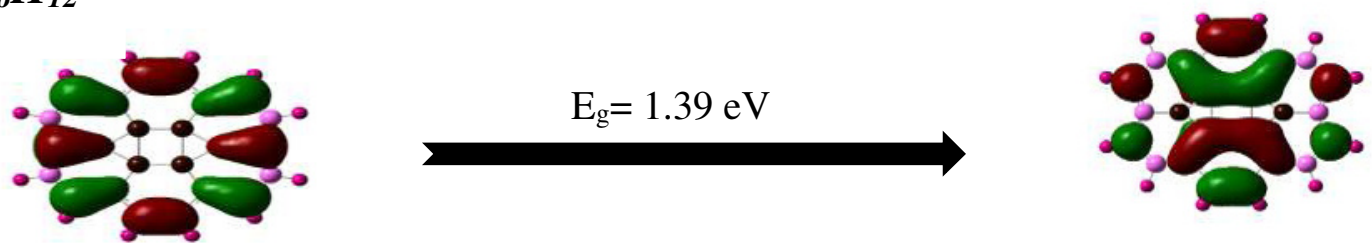

Fig 2. LUMO and HOMO molecular orbital charts of the considered atomic structures utilizing B3LYP /6-31G(d).

\section{iii) Non-Linear Optical properties (NLO)}

Nonlinear optics play an imperative part in current worldwide inquire about, as NLO dynamic materials find applications in broadcast communications, potential applications in cutting edge communication technologies, optical flag handling, and information capacity [15]. In arrange to get the connections betwe en atomic structure and nonlinear optical properties,

Table 2 displayed the values of the moment, all of which are against zero, which leads to the conviction that these values are polar particles as well. We note that these values alter with the alter of the doped the primary arrange polarizabilities and hyperpolarizabilities of coronene and their doped subordinates were calculated utilizing the DFT / B3LYP strategy with a $631 \mathrm{G}(\mathrm{d})$ basis set. It is well known that higher values of to begin with arranging dipole moment, polarizability, and hyperpolarizabilit $\mathrm{y}$ are vital for more dynamic NLO properties

administrations, which shows up clearly when the particle contains expansive esteem of around 1.56 Debye. And this is distinct from the utilitarian particle by the boron iota and with the increment 
within the number of carbon molecules, since they contain electrons on their external shell, which makes the framework more curiously, concerning polarizability $\langle\alpha\rangle$, we discover that both $\mathrm{C}_{54} \mathrm{H}_{18}$ and $C_{18} B_{6} H_{12}$ molecules have two huge values compared to other molecules, particularly the $C_{54} H_{18}$ molecule which is break even with to $101.97 \dot{A}^{3}$, all these info rmation are display within the table 3 , which we extri cated utilizing

\begin{tabular}{|c|c|c|c|c|}
\hline Compound & $\boldsymbol{\mu}(\boldsymbol{x})(\mathrm{eV})$ & $\boldsymbol{\mu}(\boldsymbol{y})(\mathrm{eV})$ & $\boldsymbol{\mu}(\mathbf{z})(\mathrm{eV})$ & $\boldsymbol{\mu}($ total) $(\mathrm{eV})$ \\
\hline $\boldsymbol{C}_{54} \boldsymbol{H}_{18}$ & 0 & 0 & 0.0006 & 0.0006 \\
\hline $\boldsymbol{C}_{24} \boldsymbol{H}_{12}$ & 0.0002 & -0.0004 & 0 & 0.0005 \\
\hline$\left[\boldsymbol{C}_{24} \boldsymbol{H}_{12} \boldsymbol{C O O H}\right]^{-}$ & -0.002 & 0 & -0.0054 & 0.0058 \\
\hline $\boldsymbol{C}_{18} \boldsymbol{N}_{6} \boldsymbol{H}_{12}$ & -1.2618 & 0.8824 & 0.1988 & 1.5525 \\
\hline $\boldsymbol{C}_{18} \boldsymbol{B}_{6} \boldsymbol{H}_{12}$ & 0.0001 & 0.0003 & -0.0009 & 0.0009 \\
\hline
\end{tabular}

Table 2. Dipolar moment (Debye units), calculated with B3LYP and correlation functionals, 6-31G (d, p)

\begin{tabular}{|c|c|c|c|c|c|c|c|}
\hline Compound & $\boldsymbol{\alpha}_{\boldsymbol{x} x}\left(\dot{A}^{3}\right)$ & $\boldsymbol{\alpha}_{y y}\left(\dot{A}^{3}\right)$ & $\boldsymbol{\alpha}_{z z}\left(\dot{A}^{3}\right)$ & $\langle\alpha\rangle\left(\dot{A}^{3}\right)$ & $<\alpha>c c$ & $\boldsymbol{r e}\left(\dot{A}^{3}\right)$ & $\Delta \boldsymbol{\alpha}\left(\dot{A}^{3}\right)$ \\
\hline $\mathrm{C}_{24} \mathrm{H}_{12}$ & 362.73 & 362.74 & 69.39 & 39.26 & 39.6 & \multirow[t]{3}{*}[16]{} & 679903.27 \\
\hline $\mathrm{C}_{54} \mathrm{H}_{18}$ & 994.69 & 979.46 & 164.76 & 101.97 & 109.3 & & 5154212.98 \\
\hline$\left[\mathrm{C}_{24} \mathrm{H}_{12} \mathrm{COOH}\right]^{-}$ & 447.48 & 414.84 & 81.70 & 46.63 & 42.5 & & 1023380.89 \\
\hline $\mathrm{C}_{18} \mathrm{~N}_{6} \mathrm{H}_{12}$ & 391.01 & 389.60 & 110.88 & 44.04 & - & \multirow[t]{2}{*}{ [17] } & 759090.72 \\
\hline $\mathrm{C}_{18} \mathrm{~B}_{6} \mathrm{H}_{12}$ & 490.66 & 397.53 & 84.93 & 48.07 & - & & 1213047.36 \\
\hline
\end{tabular}

Table 3. Average polarizability $\langle\alpha\rangle \dot{A}^{3}$ and Anisotropy $\Delta \dot{A}^{3}$, calculated with B3LYP and correlation functionals, 6-31G (d, p).

\begin{tabular}{|c|c|c|c|c|c|c|}
\hline \multicolumn{2}{|c|}{ Compound } & $\mathrm{C}_{24} \mathrm{H}_{12}$ & $\mathrm{C}_{54} \mathrm{H}_{18}$ & $\mathrm{C}_{18} \mathrm{~N}_{6} \mathrm{H}_{12}$ & {$\left[\mathrm{C}_{24} \mathrm{H}_{12} \mathrm{COOH}\right]^{-}$} & $C_{18} B_{6} H_{12}$ \\
\hline$\beta_{x x x}$ & \multirow{10}{*}{$\left(\mathcal{D} * \dot{\mathcal{A}}^{2}\right)$} & -0.0001 & 17.3021 & -0.0563 & -5.9455 & 0.0017 \\
\hline$\beta_{x y y}$ & & -0.0003 & -14.0368 & -0.0139 & -6.2278 & -0.0001 \\
\hline$\beta_{x z z}$ & & 0.0001 & 3.2171 & 0.0148 & 24.1209 & 0.0001 \\
\hline$\beta_{y y y}$ & & 0.0007 & -4.6307 & -0.0573 & 4.4265 & 0.0115 \\
\hline$\beta_{y x x}$ & & 0.0004 & -13.8737 & 0.0496 & -3.4734 & -0.0111 \\
\hline$\beta_{y z z}$ & & 0 & 2.1091 & -0.002 & 8.5727 & 0.0029 \\
\hline$\beta_{z z z}$ & & 0.0001 & 0.0003 & -0.0038 & 39.8778 & -0.0048 \\
\hline$\beta_{2 x x}$ & & -0.0077 & -0.0109 & -0.0467 & 17.9569 & -0.0086 \\
\hline$\beta_{z y y}$ & & 0.0310 & 0.0103 & -0.0335 & 7.4954 & -0.0229 \\
\hline$\beta$ & & 0.0234 & 17,6303 & 0,1011 & 67,0933 & 0,0365 \\
\hline$\beta$ & (esu) & $2.02 \times 10^{-34}$ & $1.52 \times 10^{-31}$ & $8.73 \times 10^{-34}$ & $5.79 \times 10^{-31}$ & $3.15 \times 10^{-34}$ \\
\hline
\end{tabular}

Table 4. first order hyperpolarizability $\beta m o l$ (esu), calculated with B3LYP and correlation functionals, 6-31G (d, p), with $\left(1 \mathcal{D}\right.$ ebye. $\mathcal{A n g} * * 2\left(1 \mathcal{D} * \dot{\mathcal{A}}^{2}\right)=8.6393 \times 10^{-33}$ esu $)$

The B3LYP /6-31G (d) work. For the firstorder atomic hyperpolarization $\beta$, all its information is in the table 4, we note that these values are diverse from one atom to another; the littlest esteem is the one that coronene has with a little esteem. We conver sation around $2.02 \times 10^{-34} \mathrm{esu}$, but when doped pristine with heterogeneous like nitrogen, boron, and oxygen, we take notes of an increment within the est eem of atomic hyperpolarization $\beta$, as the greatest esteem for the $\mathrm{C}_{24} \mathrm{H}_{12} \mathrm{COOH}^{-}$atom is at $5.80 \times 10^{-31}$ esu too when we increase the number of carbon particles. Consequently, we find esteem as expansive as the one we found with the C54H18 mol ecule with esteem of $1.52 \times 10^{-31}$ esu. By getting these expansive values, we accept that these molecules can be utilized in non-linear applications that have no limits by comparing the results of the average polarization rate, we found by the results 
obtained by other researchers using the same working method and the same rules, we found that the final value has great convergence. As for each of $\mathrm{C}_{18} \mathrm{~N}_{6} \mathrm{H}_{12}$ and $\mathrm{C}_{18} \mathrm{~B}_{6} \mathrm{H}_{12}$, we did not find that the researchers calculated their values related to the average polarizability

There's too something almost atomic hyperpolarizati on of the moment degree as shown in Table 5. These values are diverse from iotas constituting the particle. Within the particle $\mathrm{C}_{54} \mathrm{H}_{18}$, which has the most notew orthy esteem of $6.51 \times 10^{-36} \mathrm{esu}$, this is often due to the composition and shape of the atom, which contains an expansive number of carbon particles, taken after by the coronene particle doped with a $\mathrm{COOH}$ with the esteem of $1.7 \times 10^{-36} \mathrm{esu}$, at that point the two Coronene atoms doped with each of the Nitrogen and Boron particles, and the fundamental $\mathrm{c}$ oronene atom has the most reduced esteem with $1.3 \times$ $10^{-36}$ esu

\begin{tabular}{|c|c|c|c|c|c|c|}
\hline \multicolumn{2}{|c|}{ Compound } & $\mathrm{C}_{24} \mathrm{H}_{12}$ & $\mathrm{C}_{54} \mathrm{H}_{18}$ & $\mathrm{C}_{18} \mathrm{~N}_{6} \mathrm{H}_{12}$ & {$\left[\mathrm{C}_{24} \mathrm{H}_{12} \mathrm{COOH}\right]^{-}$} & $C_{18} B_{6} H_{12}$ \\
\hline$\gamma_{x x x}$ & \multirow{7}{*}{$\left(\mathcal{D} * \dot{\mathcal{A}}^{3}\right)$} & -3815.3034 & -18468.3101 & -4177.9022 & -4820.5588 & -4967.4342 \\
\hline $\boldsymbol{V}_{y y y y}$ & & -3815.2974 & -18166.0611 & -3472.7927 & -4403.7135 & -3247.2715 \\
\hline$Y_{z z z z}$ & & -146.7299 & -359.2025 & -238.5939 & -777.7221 & -158.7427 \\
\hline$V_{x x y y}$ & & -1271.7566 & -6075.4044 & -1246.7713 & -1550.5247 & -1389.4437 \\
\hline$V_{x x z z}$ & & -796.1257 & -3923.4718 & -881.5519 & -928.0529 & -1026.3598 \\
\hline$V_{y y z z}$ & & -796.1207 & -3832.3501 & -733.2104 & -930.5193 & -689.0756 \\
\hline $\boldsymbol{r}$ & & 2701,0673 & 12931,2053 & 2722,4712 & 3364,0376 & 2916,6413 \\
\hline $\boldsymbol{r}$ & $\left({ }^{* 10^{-36}}\right.$ esu $)$ & 1.36 & 6.51 & 1.37 & 1.69 & 1.46 \\
\hline
\end{tabular}

Table 5. second-order hyperpolarizability $\gamma$ av (*10-36 esu), calculated with B3LYP and correlation functionals, 6-31G (d, p). with $(1 \mathcal{D}$ ebye. $\mathcal{A}$.

\section{Conclusion}

In this study, we collected several different molecules based on the basic molecule of coronene $\left(C_{24} H_{12}\right)$ to determine the adequate molecule that is close to the graphene in terms of electronic, linear, and nonlinear properties. Therefore, we used the DFT method, which allows us to find each of the polarization $(\alpha)$ rate, the first hyperpolarization $(\beta)$ rate, and the second hyperpolarization $(\gamma)$ rate, and the gap energy. All these values we were extracted allow us to know what information we want and what we search for it. In brief, the linear, non-linear, and electronic properties of fundamental coronene conjointly doped with nitrogen, boron, and oxygen, our objective was to discover the ideal particle that has great properties like graphene, since it is troublesome to create it at the display time since it requires more cash and so distant there's no way to extricate a huge sum from him. The results obtained from the B3LYP theory level with $631 \mathrm{G}$ (d) basis set, appear that both $\mathrm{C}_{18} B_{6} \mathrm{H}_{12}$ and $C_{18} N_{6} H_{12}$ molecules have low bandgap values. Therefore, doped coronene have good electrical conductivity compared to undoped coronene due to their high chemical softness and low chemical hardness.

However, the relevant properties of these molecules offer several applications such as semiconductors devices, photovoltaic, as for the two $\mathrm{C}_{54} \mathrm{H}_{18}$ molecules and $\left(\mathrm{C}_{24} \mathrm{H}_{12} \mathrm{COOH}^{-}\right)$, which have a solid nonlinear optical behavior (NLO), can be utilized in (NLO) applications.

Therefore, the obtained results present the similar value as some other studies. In the case of the electronic properties, we found that $C_{18} B_{6} H_{12}$ molecule has the least gap energy with value of $1.39 \mathrm{eV}$, which is closer to the other studies. We found also, the same value as the other studies for average polarizability, especially for the $\mathrm{C}_{24} \mathrm{H}_{12}$ molecule with $39.26 \dot{A}^{3}$. 


\section{References}

[1] TY - JOUR AU - Gerstner, Ed PY - 2010 DA - 2010/11/01 TI - Nobel Prize 2010: Andre Geim \& Konstantin Novoselov JO - Nature Physics SP - 836 EP - 836 VL - 6 IS - 11 AB - The Nobel Prize in Physics 2010 has been awarded to Andre Geim and Konstantin Novoselov "for groundbreaking experiments regarding the two-dimensional material graphene".SN- 1745-2481 UR

https://doi.org/10.1038/nphys1836 DO 10.1038/nphy s1836 ID - Gerstner2010 ER

[2] Du, Yongxu \& Li, Dong \& Liu, Lb \& Gai, Guangjie. (2018). Recent Achievements of SelfHealing Graphene/Polymer Composites. Polymers. 10. 114. 10.3390/polym10020114.

[3] Shukla, Vineeta, Observation of critical magnetic behavior in 2D carbon-based composites, Nanoscale Adv., 2020,2, 962-990

[4] Santosh K. Tiwari, Sumanta Sahoo, Nannan Wang, Andrzej Huczko, Graphene research, and their outputs: Status and prospect, Advanced Materials and Devices,2020,10-29,2468-2179,

[5] Bhuyan, M.S.A., Uddin, M.N., Islam, M.M. et al. Synthesis of graphene, (2016), Int Nano Lett 6, 65-83.

[6] Tayyab, M \& H, Akhtar \& Asif, Qurat \& Syed, W. (2020). Band-gap tuning of graphene by $\mathrm{Mg}$ doping and adsorption of Brand Be on impurity: A DFT study. Computational Condensed Matter. 23. e00469. 10.1016/j.cocom. 2020.e00469.

[7] W Xinting, L Yueqing, X Wenli, Z Kaixuan, Y Jie, S Shaozhen, W Jiazhen, Di Fangfang, G Junxue, W Can, C Chaofan, Sui Ning, Chen Baoli, Zhang Yingtian, Hao Hongguo, Zhang Xianxi, Zhao Jinsheng Zhou Huawei and Wang Shuhao 2017From two-dimensional graphene oxide to three-dimensional honeycomb-like Ni3S2@graphene oxide composite: insight into the structure and electrocatalytic properties R. Soc. open sci.4171409171409http://doi.org/10.1098/rsos.17140 9.

[8] Kumar, Neeraj \& Salehiyan, Reza \& Chauke, Vongani \& Botlhoko, Orebotse \& Setshedi, Katlego \& Scriba, Manfred \& Masukume, Mike \& Sinha Ray, Suprakas. (2021). Top-down synthesis of graphene: A comprehensive review. FlatChem. 27. 100224. 10.1016/j.flatc.2021.100224.

[9] Li, N., Zhen, Z., Zhang, R. et al. Nucleation and growth dynamics of graphene grown by radio frequency plasma-enhanced chemical vapor deposition. Sci Rep 11, 6007 (2021).

https://doi.org/10.1038/s41598-021-85537-3.

[10] Heidari, Behzad \& S Ghamsari, Morteza \& Salmani, Somayeh \& Ahmadi, Mehrnoush \& MajlesAra, Mohammad. (2020). Reduced Graphene Oxide Thin Film with Strong Optical Nonlinearity. physica status solidi (b). 258. 2000397. 10.1002/pssb.202000 397

[11] Nariyangadu, S. B., Choedak, T., Malar, E. J. P., Chen, J., Thyrhaug, E., Kumar, P., Zhou, J., Yechuri, V., Pal, S. K., Lidin, S., Thangadhorai, K. N., Karki, K. J., \& Pullerits, T. (2020). New nonlinear optical crystal of rhodamine 590 acid phthalate. ACS Omega, 5(33),20863,20873. https://d oi.org/10.1021/acsomega.0c02303.

[12] Tabti, C. and Benhalima, N. (2015) Molecular Structure, Vibrational Assignments and NonLinear Optical Properties of 4,4' Dimethylaminocyan obiphenyl (DMACB) by DFT and ab Initio HF Calculations. Advances in Materials Physics and Chemistry, 5, 221-228.

http://dx.doi.org/10.4236/ampc.2015.57023.

[13] Mbala, G.F. \& Côme Damien Désiré, Mveme \& Ntieche, Z. \& Ejuh, Geh wilson \& Ndjaka, J. \& Abe, M.. (2021). Effect of chlorine and bromine on the nonlinear optical, electronic, optoelectronic and thermodynamic properties on the BEDT-TTF molecule : $\mathrm{Ab}$ initio and $\mathrm{DFT}$ calculations. 10.21203/rs.3.rs-270814/v1.

[14] Silvarajoo, Sharmili \& Osman, MD.uwaisulqar ni \& Kamarudin, Khadijah \& Razali, Mohd \& Yusoff, Hanis \& Bhat, Irshad \& Rozaini, Mohd Zul Helmi \& Juahir, Yusnita. (2020). Dataset of theoretical Molecular Electrostatic Potential (MEP), Highest Occupied Molecular Orbital-Lowest Unoccupied Molecular Orbital (HOMO-LUMO) band gap and experimental cole-cole plot of 4-(ortho, meta- and para-fluorophenyl)thiosemicarbazide isomers. Data in brief. 32. 106299. 10.1016/j.dib.202 0.106299 .

[15] Mbala, G.F. \& Abe, M. \& Ntieche, Z. \& Ejuh, Geh wilson \& Ndjaka, J.. (2021). Ab initio investigation of nonlinear optical, electronic, and thermodynamic properties of BEDT-TTF molecule: doping with boron. Heliyon. 7. e07461. 10.1016/j.hel iyon. 2021.e07461.

[16] Junqueira, G. \& Mendonça, J. \& Lima, Alessandro \& Quirino, Welber \& Sato, Fernando. (2016). Enhancement of Nonlinear Optical Properties of Graphene Oxide-based Structures: Push-Pull 
Models. RSC Advances. RSC Advances, 2016, DOI: 10.1039/C6RA18314J. 10.1039/C6RA18314J.

[17] Ejuh, Geh wilson \& Tchangnwa Nya, Fridolin \& Abe, M. \& Fankam Fankam, Jean Baptiste \& Ndjaka, J.. (2017). Electronic structure, physicochemical, linear and nonlinear optical properties analysis of coronene, 6B-, 6N-, 3B3N- substituted C24H12 using RHF, B3LYP and wB97XD methods. Optical and Quantum Electronics. 49. 10.1007/s1108 2-017-1221- 